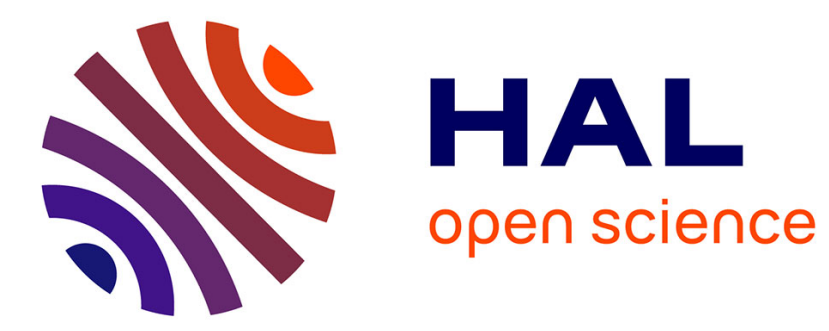

\title{
The physics of Aeolian sand transport
}

Alexandre Valance, Keld Rømer Rasmussen, Ahmed Ould El Moctar, Pascal

Dupont

\section{To cite this version:}

Alexandre Valance, Keld Rømer Rasmussen, Ahmed Ould El Moctar, Pascal Dupont. The physics of Aeolian sand transport. Comptes Rendus. Physique, 2015, 16 (1), pp.1-13. 10.1016/j.crhy.2015.01.006 . hal-01115982

\section{HAL Id: hal-01115982 \\ https://hal.science/hal-01115982}

Submitted on 24 Feb 2015

HAL is a multi-disciplinary open access archive for the deposit and dissemination of scientific research documents, whether they are published or not. The documents may come from teaching and research institutions in France or abroad, or from public or private research centers.
L'archive ouverte pluridisciplinaire HAL, est destinée au dépôt et à la diffusion de documents scientifiques de niveau recherche, publiés ou non, émanant des établissements d'enseignement et de recherche français ou étrangers, des laboratoires publics ou privés. 


\title{
The physics of Aeolian sand transport
}

\author{
Alexandre Valance ${ }^{\mathrm{a}}$, Keld Rømer Rasmussen ${ }^{\mathrm{b}}$, Ahmed Ould El Moctar ${ }^{\mathrm{c}}$, Pascal Dupont ${ }^{\mathrm{d}}$ \\ a Institut de Physique de Rennes, UMR UR1-CNRS 6251, Université de Rennes 1, 35042 Rennes cedex, France \\ ${ }^{b}$ Geoscience, Aarhus University, Denmark \\ ${ }^{c}$ Laboratoire de Thermocinétique, Polytech. Nantes, CNRS UMR 6607, 44306 Nantes, France \\ ${ }^{d}$ LGCGM, INSA de Rennes, Campus Beaulieu, 35043 Rennes, France
}

\begin{abstract}
We give a synthetic overview of the state of art of the physics of sand aeolian transport. We first present the main ideas developed by Bagnold in the middle of the last century. We then review the recent experimental and theoretical advances made in the field and emphasize that the particle flow rate does not exhibit a cubic dependence with the air friction speed, as predicted by Bagnold, but a quadratic one. Finally, we list important open issues that remain.
\end{abstract}

\section{Résumé}

Nous donnons un aperçu synthétique de l'état de l'art sur la physique du transport éolien du sable. Nous présentons d'abord les principales idées développées par Bagnold au milieu du siècle dernier. Puis nous exposons les avancées récentes dans le domaine tant du point de vue expérimental que théorique. Nous insistons en particulier sur le fait que le flux particulaire ne suit pas une loi cubique avec la vitesse de frottement de l'air, comme prédit par Bagnold, mais une loi quadratique. Nous listons enfin les questions importantes qui restent à traiter.

Keywords: Aeolian sand transport; Saltation; Particle-laden fluid flows;

Mots-clés : Transport éolien du sable; Saltation; écoulements fluides chargés de particules;

\section{Introduction}

A major advance in the study of wind-blown sand started seventy years ago with the seminal work of Bagnold (Bagnold, 1941). Since then and in particular in the last decades, important progress has been made thanks to the developments of efficient imaging velocimetry techniques (e.g., PIV and PTV) and numerical simulations based on discrete elements methods (D.E.M). The objective of the present paper is to give a synthetic review of recent developments of the physics of aeolian sand transport for a broad audience of physicists. Readers willing to go deeper in the details, we refer to the recent extensive reviews from Duran et al. (2011) and Kok et al. (2012).

The paper is organized as follows. The second section presents the main ideas developed by Bagnold. The third section reports recent experimental findings drawn from wind-tunnel experiments while the fourth section focuses on the physical processes occurring close to the bed surface when a grain impacts with the bed, rebounds and ejects other grains. Section 5 presents theoretical and numerical approaches while Section 6 briefly discusses sand transport dynamics for unsteady and inhomogeneous conditions. Finally, the last section lists some important open issues in aeolian sand transport. 


\section{Bagnold picture}

We present here the main important concepts and ideas developed by Bagnold in his seminal work which dates from 1941 (Bagnold, 1941). Bagnold was the first to identify the dominant mode of sand transport, termed saltation, which consists of grains traveling in a sequence of ballistic trajectories and producing a splash of new ejected grains (termed reptation grains) when colliding back onto the soil (see Figure 1).

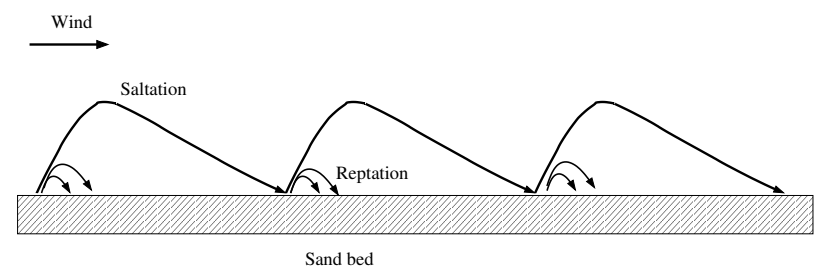

Figure 1: Schematic of the different modes of aeolian sand transport: (i) saltation mode and (ii) reptation mode.

To initiate saltation transport, the wind speed should exceed a certain velocity threshold, referred hereafter to as the static threshold. To have an estimate of the static threshold, one can write a simple balance of the forces acting on a grain located at the bed surface. Neglecting cohesion, the grain is subject to two competitive forces: (i) a driving drag force, $F_{d} \approx \alpha\left(\pi d^{2} / 4\right) s_{a}$ (where $d$ is the grain diameter, $s_{a}$ is the air borne shear stress at the bed surface and $\alpha$ is a constant parameter depending on the details of the drag force; see for example Duran et al. (2011)), and (ii) a resistive force induced by contact friction, $F_{\text {friction }} \approx(\pi / 6) \mu\left(\rho_{a}-\rho_{f}\right) g d^{3}$, (where $\mu$ is the solid friction coefficient and $\rho_{p}$ and $\rho_{a}$ are the grain and air density respectively). The force balance yields

$$
\frac{s_{a}}{\left(\rho_{p}-\rho_{a}\right) g d} \approx \frac{2 \mu}{3 \alpha} \text {. }
$$

This relation tells us that the threshold for incipient motion corresponds to a finite value of the ratio of the basal shear stress to the effective grain weight, usually referred to as the Shields number $S^{*}$. For sand grains with a diameter greater than $100 \mu \mathrm{m}^{1}$, the static threshold Shields number is of the order of $S_{s}^{*} \approx 0.01$.

In addition to the static threshold, Bagnold identified a second threshold, referred to as the dynamic one, $S_{d}^{*}$, corresponding to the critical wind strength at which saltation ceases once initiated. The dynamic threshold is smaller than the static one $\left(S_{d}^{*} \approx 0.006\right)$ and is due to the fact that once transport has been initiated, erosion induced by the impact of saltating grains on the bed - the splash - is a very efficient mechanism to sustain saltation transport.

As saltation is initiated, the number of saltating grains first increases exponentially due to the multiplicative process of the splash events (Anderson et al., 1991). Wind loses momentum to accelerate the grains and the flux eventually saturates after a certain distance (Sauermann et al., 2001; Andreotti et al., 2010; Pähtz et al., 2013). In this equilibrium or saturated state, the transport rate, $Q$, can be expressed by virtue of momentum conservation (Sørensen, 2004):

$$
Q=\frac{l_{s}}{u_{0 \downarrow}-u_{0 \uparrow}}\left(s_{a \infty}-s_{a 0}\right)=\frac{l_{s}}{u_{0 \downarrow}-u_{0 \uparrow}} \rho_{a}\left(u^{* 2}-u_{0}^{* 2}\right),
$$

\footnotetext{
${ }^{1}$ For fine grains typically smaller than $100 \mu \mathrm{m}$, cohesion forces come into play and are responsible for increasing the threshold at small $d$ (see for example (Claudin and Andreotti, 2006))
} 
where $l_{s}$ is the mean saltation length (to be understood as the hoping length averaged over the particles), and $u_{0 \downarrow}$ (resp. $u_{0 \uparrow}$ ) is the mean horizontal velocity of saltating grains before they collide the bed (resp. when they leave the bed). $s_{a \infty}$ is the air borne shear stress far above the transport layer and defines the air friction speed, $u^{*}=\sqrt{s_{a \infty} / \rho_{a}}$, and the Shields number, $S^{*}=s_{a \infty} /\left(\rho_{p}-\rho_{a}\right) g d$, of the flow. $s_{a 0}$ is the residual air borne shear stress at the bed in the equilibrium state of transport and $u_{0}^{*}$ is the corresponding basal friction velocity $\left(u_{0}^{*}=\sqrt{s_{a 0} / \rho_{a}}\right)$. Eq. (2) emphasizes that the dependence of the saltation length and particle velocity on wind strength (measured through $S^{*}$ or $u^{*}$ ) is a key issue to derive a scaling relation for $Q_{s a t}$ as a function of the air friction speed $u^{*}$.

Bagnold was the first to derive a scaling law for $Q$. Using arguments based on dimensional analysis, he assumed that the saltation length scales as $u^{* 2}$ and the particle velocity as $u^{*}$. He also hypothesized that the air borne shear stress at the bed in the equilibrium state of transport is reduced to zero. With these assumptions, Eq. (2) yields the famous Bagnold law with a cubic dependence on $u^{*}$ (Bagnold, 1941):

$$
Q=A_{B} \frac{\rho_{a}}{g} u^{* 3} .
$$

However, recent wind-tunnel experiments and numerical models led us to reconsider Bagnold's assumptions. In particular, the saltation length and particle velocity are actually independent of the wind strength, leading to a quadratic scaling for the transport law instead of the cubic one found by Bagnold. Besides, numerical models indicate that the residual basal shear stress is equal to the dynamical threshold Shields number $\left(S_{d}^{*}\right)$ for moderate winds but vanishes for very strong winds (see Section 5).
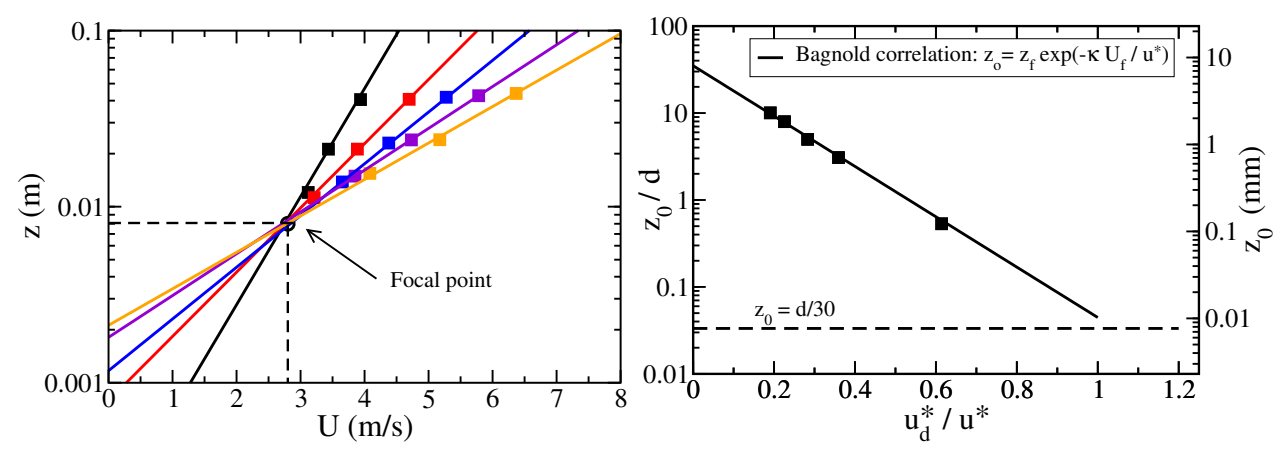

Figure 2: (a) Air velocity profiles obtained with natural sand of median diameter $d=230 \mu \mathrm{m}$ for various wind strengths. $\left(u^{*}=0.28,0.47,0.60,0.75,0.89 \mathrm{~m} / \mathrm{s}\right)(\mathrm{Ho}, 2012)$; (b) Aerodynamic roughness length $z_{0}$ as a function of $u_{d}^{*} / u^{*}(\mathrm{Ho}$, 2012)

Bagnold evidenced another salient feature of aeolian sand transport by identifying a critical height, $z_{f}$, where the air velocity is independent of the wind shear stress: $U\left(z_{f}\right)=U_{f}$. This critical location was termed later the Bagnold focus point. Extensive wind-tunnel experiments by Iversen and Rasmussen (1999) indicate that the wind speed $U_{f}$ at the focus point scales linearly with the dynamic threshold velocity, $u_{d}^{*}=\sqrt{(\sigma-1) g d S_{d}^{*}}$ (where $\left.\sigma=\rho_{p} / \rho_{a}\right)$, whereas the focus height $z_{f}$ is on the order of a centimeter and varies roughly linearly with the grain diameter. For $z>z_{f}$, Bagnold shows that the wind velocity profile exhibits a logarithmic profile,

$$
U(z)=\frac{u^{*}}{3} \ln \frac{z}{z_{0}},
$$


with an effective aerodynamic roughness length, $z_{0}$, which depends on the transport strength (see Fig. 2). Using the continuity of the velocity profile at the focus point (i.e., $\left.U_{f}=\left(u^{*} / \kappa\right) \ln \left(z_{f} / z_{0}\right)\right)$, the effective roughness length can be expressed as:

$$
z_{0}=z_{f} \exp \left(\frac{-\kappa U_{f}}{u^{*}}\right) .
$$

$z_{0}$ is an increasing function of the friction velocity. This is a clear signature of the presence of particles within the flow which strongly interact with it. This result indeed contrasts with the situation without transport (i.e., below the dynamical threshold), where the aerodynamic roughness length is independent of the wind strength and is given by the geometrical bed roughness $\left(z_{0} \approx d / 30\right)$ in the rough turbulent flow regime. Other expressions for the effective aerodynamic roughness have been proposed in the literature, as the one from Owen (1964) (i.e., $z_{0} \propto u_{*}^{2} / g$ ), that are also supported by the experimental data (Sherman, 1992; Rasmussen et al., 1996).

It will be argued below that the Bagnold focus point can be interpreted as the characteristic height of the transport layer where the majority of the particle are transported and substantially slow down the flow. Besides, as to be discussed later on in Section 5, theoretical and numerical models (Anderson and Haff, 1988, 1991) indicate that below the focus point, the air velocity is almost invariant with the friction velocity $u^{*}$.

\section{Recent wind-tunnel development}

Since Bagnold seminal work and particularly within the two last decade, a multitude of field and wind-tunnel studies (Willetts and Rice, 1986, 1989; Nalpanis et al., 1993; Greeley et al., 1996; Namikas et al., 2003; Dong et al., 2004; Dong and Wang, 2004; Rasmussen and Sørensen, 2008; Creyssels et al., 2009; Ho et al., 2011, 2012) have been carried out to refine the understanding of the process aeolian transport. Thanks to the improvement of imaging techniques [e.g. Particle Image Velocimetry (PIV) and Particle Tracking Velocimetry (PTV) (Creyssels et al., 2009)], it is possible now to get a more accurate description of the saltation cloud. In particular, recent wind-tunnel experiments (Dong et al., 2004; Dong and Wang, 2004; Rasmussen and Sørensen, 2008; Creyssels et al., 2009) allowed to provide a rather accurate characterization of the aeolian transport in terms of particle velocity and concentration within the saltation layer. The most salient features drawn from these recent experiments are summarized below.

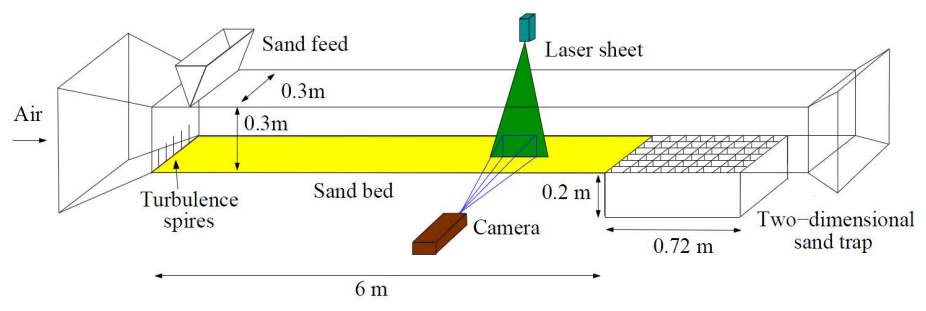

Figure 3: Wind tunnel used by Ho et al. (2011) for characterizing the saltation transport using Particle Image Velocimetry and sand trap system. 


\subsection{Particle velocity}

Recent experiments (Ho, 2012) provide accurate measurements of the averaged particle velocity as a function of the height from the bed (see Fig. 4.a). These profiles clearly indicate a crossover between a layer close to the bed and above. Within this layer whose depth roughly corresponds to the Bagnold focus height, the particle velocity profile is found almost insensitive to the wind shear stress in contradiction with Bagnold's hypothesis stating that the latter scales linearly with the friction velocity. More specifically, for $z<z_{f}$, the average horizontal particle velocity ${ }^{2}$

$$
u(z)=u_{0}+\gamma z,
$$

where the average slip velocity $u_{0}$ and the shear rate $\gamma$ are independent of the wind strength Wind-tunnel experiments from Ho (2012) with quartz sand of median diameter $d=230 \mu \mathrm{m}$ show that $u_{0} \approx 15 \sqrt{g d}$ and $\gamma \approx 0.5 \sqrt{g / d}$ (see Fig. 4a). In contrast, the average vertical particle
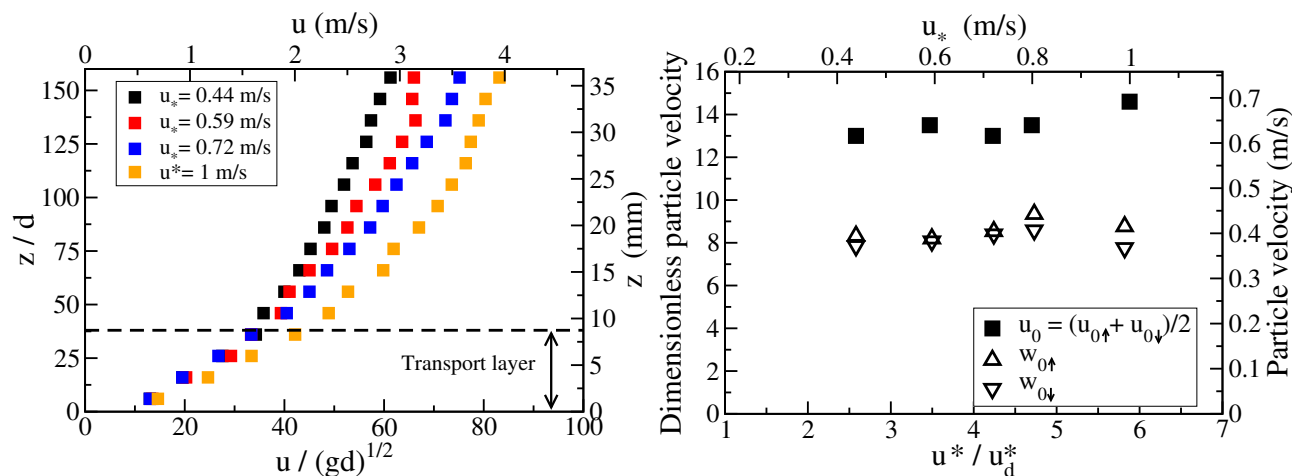

Figure 4: (a) Average particle velocity profiles obtained with natural sand of median diameter $d=230 \mu \mathrm{m}$ for various wind strengths. $\left(u^{*}=0.44,0.59,0.72,1 \mathrm{~m} / \mathrm{s}\right)(\mathrm{Ho}, 2012)$; (b) Average horizontal slip velocity $u_{0}$ and average vertical velocity of upward and downward particles close to the bed surface ( $w_{0 \uparrow}$ and $\left.w_{0 \downarrow}\right)$ versus the friction velocity (Ho, 2012)

velocity is reduced to zero in the equilibrium state of transport, but the average vertical velocity of downward and upward particles respectively are finite and opposite (see Fig. 4b).

This layer close to the bed (and bounded by the Bagnold focus height), termed hereafter saltation or transport layer, can be identified as the region where the majority of the particles are transported. Specifically, more than $75 \%$ of the total mass flux is transported within this layer (Ho, 2012). Particle concentration is so high that the air flow is strongly slowed down. An increase of the wind strength results in an increase of the particle concentration which in turn leads to a decrease of the air flow speed close to the bed such that the new equilibrium particle velocity remains almost unchanged. In contrast, above the saltation layer, the particle concentration is much less and the air flow is almost unaffected by the presence of the particle. As a result, the particle velocity above the saltation layer increases with increasing wind strength.

Another important feature is that the particles present large velocity fluctuations. Recent experiments (Greeley et al., 1996; Namikas et al., 2003; Ho et al., 2012) show that saltating

\footnotetext{
${ }^{2} u(z)$ and $w(z)$ denote the horizontal and vertical components of the average Eulerian particle velocity at the height $z$. The average is taken over all particles located at the height $z$. Further in the article, when referring to the velocity components of a given particle, we use the variables $\xi_{x}$ and $\xi_{z}$, respectively.
} 
particles exhibit non-Gaussian velocity distributions with flat tails. Typical vertical velocity distributions obtained from wind-tunnel experiments (Ho et al., 2012) and measured close to the bed surface are shown in Fig. 5. These distributions exhibit unambiguously a deviation from a Gaussian distribution. A long-tail is clearly observed. Moreover, the vertical velocity distributions within the transport layer do not significantly change with the wind strength confirming that the features of the saltating particles within the saltation layer are invariant with the Shields number (Ho et al., 2011). The same trend is observed for the horizontal particle distribution (i.e., flat tail and invariance with the wind strength).

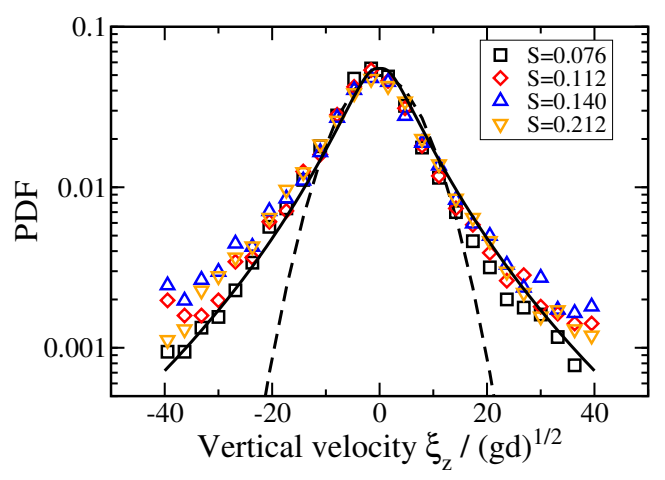

Figure 5: Vertical velocity distribution $p\left(\xi_{z}\right)$ of saltating particles located close to the bed surface within the transport layer (at $z=10 d$ ) for various wind strengths obtained from wind-tunnel experiments (Ho et al., 2012). Fitted distributions: (dashed line) Gaussian law with variance $s^{2}=53 \mathrm{gd}$ and (solid line) lognormal law.

\subsection{Particle concentration}

Another important feature characterizing the saltation transport is the particle concentration. Experiments (Rasmussen and Sørensen, 2008; Creyssels et al., 2009; Ho, 2012) indicate that the particle volume fraction decreases within increasing height and its magnitude increases with increasing wind strength. More precisely, the particle volume fraction is found to scale linearly with the Shields parameter as:

$$
\phi(z) \propto\left(S^{*}-S_{d}^{*}\right) f\left(z / l_{\phi}\right)
$$

where $f$ is a decreasing function of $z$, which asymptotically approaches an exponential behavior at large height (see Fig. 6): $f\left(z / l_{\phi}\right) \approx e^{-z / l_{\phi}}$ for $z>z_{f}$. $l_{\phi}$ is the characteristic decay height of the particle concentration and is found to be invariant with the wind strength and is of the same oder of magnitude as the focus height $z_{f}$. This confirms that the focus height is intimately related to the vertical extension of the saltation layer. Interestingly, experimental data (see Fig. 6) show that the particle volume fraction clearly deviate from the exponential behavior within the saltation layer. This deviation may be attributed to the non-Gaussian velocity distribution of the saltating particles.

As a partial conclusion, the important picture to keep in mind is that an increase of wind speed essentially leads to an increase of the particle concentration leaving the particle velocity almost unchanged within the transport layer. The vertical extent of the transport layer is also independent of the wind strength. This last feature is fully correlated with the existence of the Bagnold focus point. 


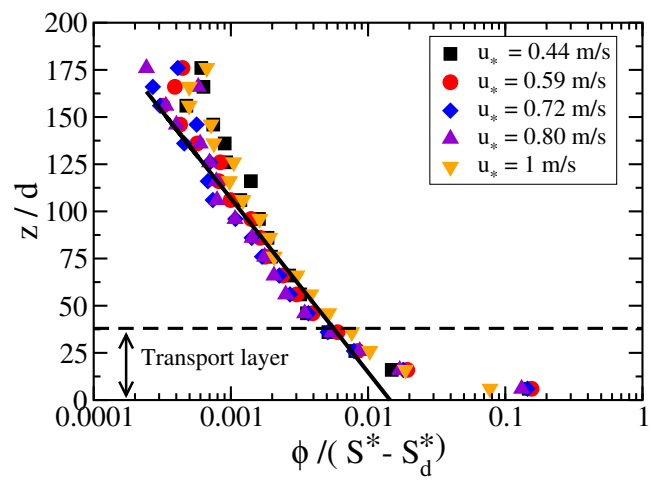

Figure 6: (a) Particle volume fraction profiles rescaled by $\left(S^{*}-S_{d}^{*}\right)$ obtained with natural sand of median diameter $d=230 \mu \mathrm{m}$ for various wind strengths $\left(u^{\star}=0.44,0.59,0.72,0,80,1 \mathrm{~m} / \mathrm{s}\right)$ (Wind-tunnel experiments by Ho (2012)).

\subsection{Horizontal and Vertical particle fluxes}

An important consequence of the above results is that the mass transport rate $(Q)$ in the equilibrium state, which can be obtained by the vertical integration of the product of the particle velocity, $u(z)$, and the particle volume fraction, $\phi(z)$, scales linearly with the Shields parameter or quadratically with the shear velocity (Ho et al., 2011):

$$
Q=A_{H} \rho_{p} d \sqrt{g d}\left(S^{*}-S_{d}^{*}\right)=A_{H} \sqrt{\frac{d}{g}} \rho_{a}\left(u^{* 2}-u_{d}^{* 2}\right),
$$

where $A_{H}$ is a constant which may depend on the mechanical properties of the grains and also on their size. This quadratic scaling has been suggested first by (Ungar and Haff, 1987) from a numerical model and confirmed later by more refined numerical and theoretical approaches (Andreotti, 2004; Creyssels et al., 2009; Duran et al., 2012) (see Section 5 for further details). This result contrasts with the Bagnold cubic scaling which is still widely misused in aeolian studies. The quadratic scaling is explained by the fact that the particle velocity within the saltation layer (and consequently the mean saltation length) is almost insensitive to the wind strength as seen earlier. These features when combined with Eq. 2 lead to a quadratic scaling with the air friction velocity. The Bagnold cubic scaling seems however to hold either at very large wind strength (i.e., $u^{*}>5 u_{d}^{*}$ ) (Duran et al., 2011) or for sand transport over rigid beds (Ho et al., 2011; Jenkins and Valance, 2014). Such a scaling corresponds to a situation where the transport layer expands with increasing wind speed and is so dilute that the air flow is barely affected by the presence of the particles.

The vertical mass flux at the bed, $\Phi_{0}$, is the erosion rate. $\Phi_{0}$ is obtained by the product of the particle volume fraction and the vertical velocity of the ascending (or descending) particles at the bed and scales linearly with the Shields parameter (Ho, 2012):

$$
\Phi_{0}=B_{H} \rho_{p} \sqrt{g d}\left(S^{*}-S_{d}^{*}\right)=B_{H} \frac{\rho_{a}}{\sqrt{g d}}\left(u^{* 2}-u_{d}^{* 2}\right),
$$

where $B_{H}$ is a constant which depends on the material properties of the grains. The transport rate $Q$ and the vertical flux $\Phi_{0}$ at the bed surface are closely connected. One can indeed show (Ho et al., 2014) that

$$
Q=\overline{l_{s}} \Phi,
$$




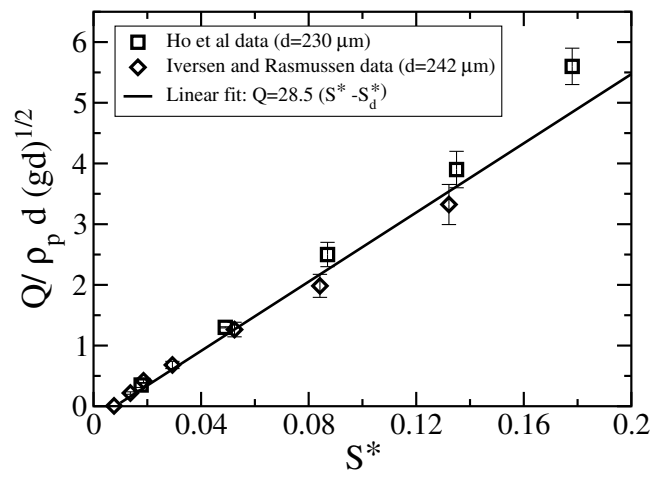

Figure 7: Mass flow rate versus the Shields parameters obtained from wind-tunnel experiments with sand grains of mean diameter $d=230 \mu m$ (Ho et al., 2011) and $d=242 \mu m$ (Iversen and Rasmussen, 1999), respectively.

where $\overline{l_{s}}$ is the average hop length of the saltation particles. Combining the above results (cf. Eqs 8-9), we arrive at the conclusion that the mean saltation length $\overline{l_{s}}$ should be invariant with the wind strength as evidenced by indirect measurements in Ho et al. (2011).

\subsection{Saltation trajectory and length}

The above scaling laws were obtained from Eulerian approaches (through PTV or PIV techniques). With these techniques one can only assess local quantities such as particle velocity and concentration. Lagrangian approaches aiming at characterizing the trajectory of saltating particles are much less numerous (White and Schulz, 1977; Willetts and Rice, 1988; Nalpanis et al., 1993; Rice et al., 1995, 1996; Zhang et al., 2007) because they are subject to strong operational requirements (Ho et al., 2014). These requirements impose to work at moderate wind shear stress (i.e., close to the transport threshold) where the particle concentration within the transport layer is sufficiently low.

An alternative method has been developed to assess the distribution of the saltation length based on classical sand trap devices (Ho et al., 2014). This method consists in using a simple sand trap device using a two-dimensional array of vertical bins located at the downwind end of a wind-tunnel (see Fig. 3). From the distribution of the mass of sand collected in the bins along the stream-wise direction $(x)$, it is possible to extract the vertical mass flux $\Phi(x)$. The latter quantity is intimately connected to the hop length distribution and can be expressed as

$$
\Phi(x)=\Phi_{0} \int_{x}^{+\infty} P\left(l_{s}\right) d l_{s} .
$$

where $P\left(l_{s}\right)$ is the probability distribution function of the hop length. Eq. 11 tells us that the mass trapped at the position $x$ comes from the contribution of saltating particles having a hopping length greater than $x$. From Eq. 11, it is straightforward to show that the hop length distribution $P\left(l_{s}\right)$ and the average of hop length are given respectively by:

$$
P_{l}(x)=-\frac{1}{\Phi_{0}} \frac{d \Phi}{d x},
$$

and

$$
\overline{l_{s}}=\int_{0}^{+\infty} l_{s} P\left(l_{s}\right) d l_{s}=\frac{Q_{s a t}}{\Phi_{0}} .
$$


An example of vertical flux distributions $\Phi(x)$ obtained from sand trap measurements for various Shields parameters (Ho et al., 2014) is shown in Fig. 8. These distributions strongly deviate from an exponential law and exhibit flat tails, which are reminiscent of the particle velocity distribution. Most importantly, the normalized vertical flux distribution $\Phi(x) / Q$ is weakly sensitive to variation of the flow strength. It thus results that the mean saltation length is invariant with the Shields parameters (see Fig. 8b). Theses experimental outcomes provide a direct confirmation of the invariance of the mean saltation length with the wind strength.

This feature results from the strong coupling between the air flow and the sand particles within the transport layer. As already mentioned, an increase of the wind strength essentially leads to an augmentation of the particle concentration but leaves the particle speed almost unchanged. The particle velocity within the saltation layer is primarily governed by the collision process between the saltating particles and the sand bed. More precisely, the impacting velocity adjusts its magnitude such that the average replacement capacity (i.e., the number of grains set into motion by a collision event, including the rebound particle and the grains expelled from the bed) falls down to one (Anderson and Haff, 1991; Creyssels et al., 2009; Duran et al., 2011). As to be seen in the next section, this occurs at a critical impacting velocity which depends only on the mechanical properties of the sand grain and scales as $\sqrt{g d}$ (Beladjine et al., 2007). In the equilibrium state of transport, the particle speed within the saltation layer and consequently the saltation hop are thus invariant with the wind strength.
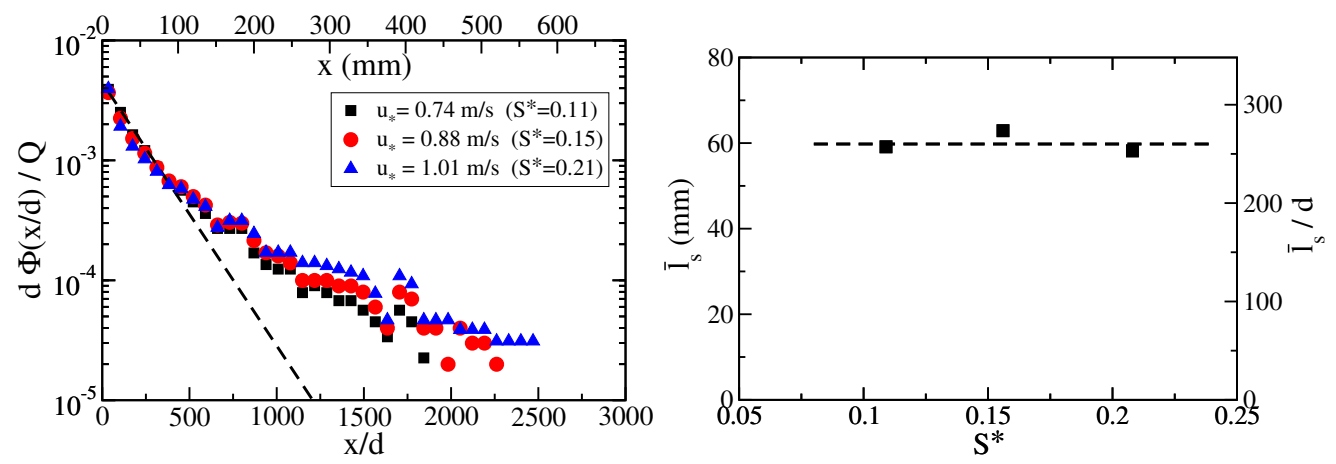

Figure 8: (a) Vertical mass flux distribution $d \Phi(x) / Q$ for various Shields parameters (experimental data by Ho et al. (2014) with $230 \mu \mathrm{m}$ sand). Note that we have normalized the distribution $\Phi(x)$ by the mass flow rate $Q$ such that $\int_{0}^{\infty} d x \Phi(x) / Q=1$. The dash line represents the best fit to the data obtained with an exponential law. (b) Dimensionless saltation length $\bar{l} / d$ versus the Shields (experimental data by Ho et al. (2014) with $230 \mu m$ sand.

\section{Splash process}

The splash process is the process in which a grain impacts the bed, rebounds, and ejects other grains. The description of this process is a key ingredient for theoretical and numerical approaches as to be seen in the next section. Because of the large concentration and velocity gradients very near the bed in a steady state of saltation, it is difficult to make reliable measurements on the splash process in situ, i.e., in wind tunnels and this is a challenge for future experiments. As a consequence, the splash process has been analyzed under somewhat artificial conditions: in computer simulations (Werner and Haff, 1988; Anderson and Haff, 1991; Oger et al., 2005), in wind tunnel experiments with very small transport rates (Willetts and Rice, 1989; Rice et al., 
1996), and in model collision experiments consisting in propelling a single particle (Mitha et al., 1986; Rioual et al., 2000; Beladjine et al., 2007) or sand grain (Werner, 1990) into a static bed of similar particles.

Two distinct outputs can be identified after a collision of particle onto a granular bed (as illustrated in Fig. 9): the rebound of the impact particle and a set of the splashed particles. On
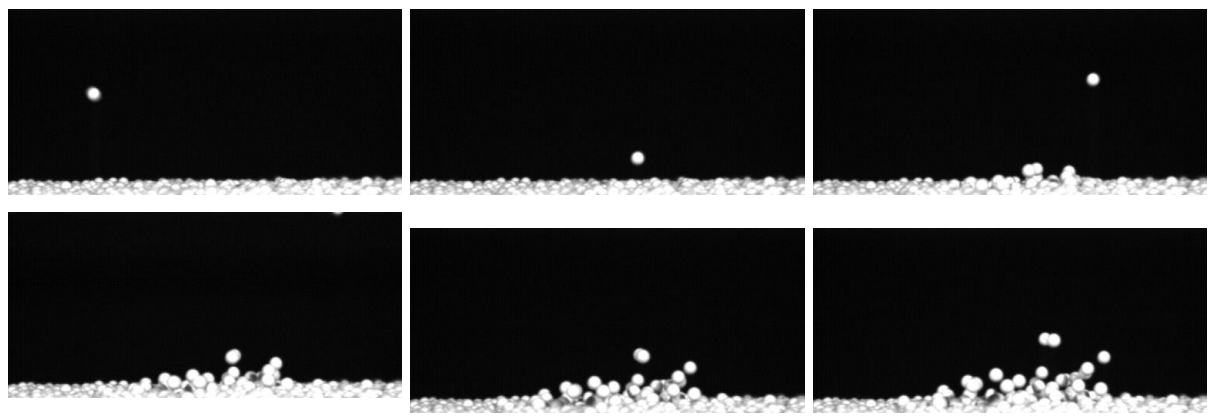

Figure 9: Successive Snapshots of a collision of a $6 \mathrm{~mm}$ PVC bead onto a granular packing of like particles (Beladjine et al., 2007). The impact velocity and angle are, respectively, $\xi_{i}=74 \sqrt{g d}=18 \mathrm{~m} / \mathrm{s}$ and $\theta_{i}=40^{\circ}$ and the collision. The time step between two successive images is $4 \mathrm{~ms}$.

the basis of the model collision experiments, important features both for the rebound particle and the splashed particles have been identified and are summarized below. Numerical simulations based on discrete element method (D.E.M) (Werner and Haff, 1988; Anderson and Haff, 1988, 1991; Oger et al., 2005; Crassous et al., 2007) generally confirm the experimental outcomes.

\subsection{Rebound}

An important feature of the rebound process is that the latter is not specular for grazing impact angles $\left(\theta_{r} \neq \theta_{i}\right)$ The model collision experiments from Beladjine et al. (2007) show that at grazing angles, the rebound angle is greater than the incident angle, whereas for impact angles higher than $20^{\circ}$, the rebound angle is less than the incident one. A consequence of this is that there is a conversion of the horizontal momentum of the impacting particle into the vertical momentum of the rebound particle. This feature is clearly seen through the vertical restitution coefficient $e_{z}$ characterizing the rebound and defined as $e_{z}=\xi_{z, r} / \xi_{z, i}$ (where $\xi_{z, r}$ and $\xi_{z, i}$ are, respectively, the vertical components of the rebounding and impacting velocity). As shown in Fig. 10a, $e_{z}$ increases with decreasing impact angle and exhibits values greater than 1 for impact angle smaller than $20^{\circ}$. This conversion from the horizontal to the vertical direction is essential in order to maintain the saltation motion. Indeed, a saltating particle in a steady motion has to reach on average the same height for each of its successive jumps. During the ascending phase of the jump, the saltating particle experiences vertical air drag forces, thus dissipating energy. The energy dissipation in the vertical direction should be balanced by energy gain. This balance is made possible thanks to the momentum conversion during the collision.

To describe completely the rebound process, it is convenient to introduce a second restitution coefficient, $e$, defined as the ratio of the rebound velocity to that of the impact velocity: $e=\xi_{r} / \xi_{i}$. The knowledge of both coefficients, $e_{z}$ and $e$, is sufficient to characterize the rebound process. As shown in Beladjine et al. (2007), both are independent of the impact velocity and are decreasing 

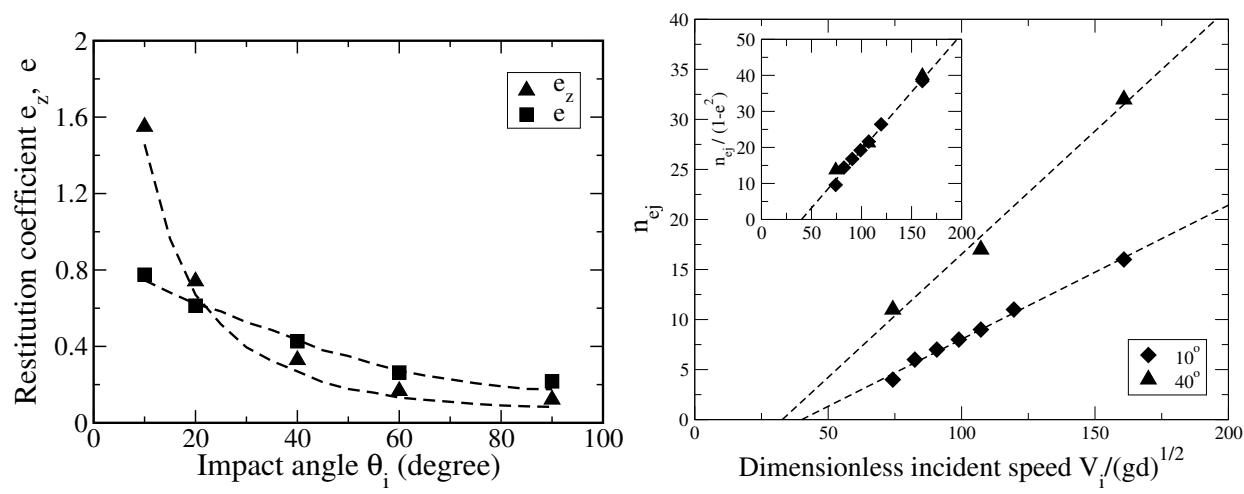

Figure 10: Experimental data from model collision experiments with $6 \mathrm{~mm}$ PVC beads (Beladjine et al., 2007): (a) Variation of the average restitution coefficient $e_{z}$ and $e$ as a function of the impact angle $\theta_{i}$ for a fixed impact velocity $\xi_{i}=106 \sqrt{g d}=26 \mathrm{~m} / \mathrm{s}$. The dotted lines stand for best fits of the form: $A_{z} / \sin \theta_{i}-B_{z}$ for $e_{z}$ (with $A_{z}=0.30$ and $B_{z}=0.15$ ), and $A-B \sin \theta_{i}$ for $e$ (with $A=0.87$ and $B=0.72$ ). (b) Mean number of ejected beads versus the impact speed for two different impact angles $\theta_{i}=10^{\circ}(\diamond)$ and $40^{\circ}(\boldsymbol{\Delta})$.

functions of the impact angle. They can be well approximated by the following laws:

$$
e_{z} \approx \frac{a_{z}}{\sin \theta_{i}}-b_{z}, \text { and } e \approx a-b \sin \theta_{i} .
$$

$a_{z}, b_{z}, a$ and $b$ are constants, which are expected to depend on the material properties of the particles. As shown in Crassous et al. (2007), the key material property is the dissipation rate in a binary collision and can be characterized by the normal restitution coefficient $\varepsilon$ within a binary collision. The plastic particles used in the model experiments of Beladjine et al. (2007) have a normal restitution coefficient close to that found with glass beads (which are expected to behave as quartz sand grains): $\varepsilon=0.9$. For these particles, the constants in Eq. 14 have the following values: $a_{z}=0.30, b_{z}=0.15, a=0.87$, and $b=0.72$. One can mention that Werner and Haff (1988) proposed the same type of law for $e_{z}$, deduced from a model collision experiment achieved with sand grain. They found slightly different values but compatible with those of Beladjine et al. (2007): $a_{z}=0.26$ and $b_{z}=0.19$.

\subsection{Splashed particles}

When the incident bead impacts the bed, it does not only rebound but can also eject other beads which fly off in all directions with a speed which rarely overcomes $10 \%$ of the incident velocity. The number of splashed particles at a given impact angle increases linearly with the impact speed above a critical velocity $\xi_{c}$ (Beladjine et al., 2007) (see Fig 10b):

$$
n_{e j}=f_{e j}\left(\theta_{i}\right)\left[\frac{\xi_{i}-\xi_{c}}{\sqrt{g d}}\right],
$$

where $f_{e j}\left(\theta_{i}\right)$ is an increasing function of the impact angle and $\xi_{c}$ is the critical velocity below which there is no splashed particles. This critical velocity is roughly independent of the impact angle (Werner and Haff, 1988; Beladjine et al., 2007). Werner and Haff (1988) found that $\xi_{c} \approx$ $20 \sqrt{g d}$, whilst Beladjine et al. (2007) obtained a significantly greater value, $\xi_{c} \approx 40 \sqrt{g d}$. Note 
also that for very weak impacting velocity (i.e., $\xi<2 \sqrt{g d}$ ), the incident particle is not able to rebound; it is trapped by the particle bed.

The existence of a critical velocity for producing splashed particles is an important feature of the splash process. As a matter of fact, the critical impact velocity $\xi_{c}$ sets the velocity of the saltating particles in the equilibrium state of transport. At equilibrium, the net production of the splashed particles should vanish (if not the particle concentration in the transport layer would increase indefinitely). This requirement imposes that the average impact velocity of the saltating particles should not exceed $\xi_{c}$. Theoretical models of transport, as the one developed by Creyssels et al. (2009), show that the average particle velocity at impact, $u_{0 \downarrow}$ is about half of $\xi_{c}$.

The linear dependence of the number of splashed particles with the impact velocity indicates that the splash process is predominantly driven by the transport of momentum rather than energy. A collision process based on energy transport would have lead to a quadratic dependence of the splashed particles with the impact velocity: $n_{e j} \propto \xi_{i}^{2} / g d$.

Several authors propose a functional form for the dependency of the number of splashed particles with the impact angle. Werner and Haff (1988) proposes a simple function: $f_{e j} \approx$ $3 \sin \theta_{i}$; while Beladjine et al. (2007) thanks to extensive collision experiments shows that the function $f_{n e j}$ is strongly correlated to the energy loss of the impacting particle during the rebound: $f_{e j} \approx 13\left(1-e^{2}\right)$. The deep reason for this correlation is not completely understood. Based on momentum conversation, we would have expect instead that $f_{e j} \propto(1-e)$. This is however not what is found based on experiments.

To complete the description the splashed process, one has to specify, in addition to the number of splashed particles, the take-off velocity and angle of the splashed particles. The model collision experiments show that the average take-off velocity of the splashed particles is independent of the impact angle and weakly sensitive to the impact speed. It results that the distribution of the take-off velocity is also independent of the impact angle and weakly sensitive to the impact speed. Its main feature is that it exhibits a large tail. Several distribution laws have been proposed such as the exponential function, the gamma function and the lognormal distribution. All these distributions are good candidates to describe the experimental data and it is difficult to discriminate between them. Among these distributions, one can mention the Raleigh distribution proposed by Werner and Haff (1988) and the lognormal distribution suggested by Beladjine et al. (2007).

The average take-off angle of the splashed particles is found to be independent of the impact speed and weakly dependent of the impact angle Beladjine et al. (2007). It varies from $80^{\circ}$ for grazing impact angles to $90^{\circ}$ for normal impact. The distribution function of the take-off angle, $P(\theta)$, was shown to be well approximated by a circular normal distribution Beladjine et al. (2007) with a variance $s^{2}$ which is almost invariant with the impact velocity and angle $(s \approx \pi / 8)$. Finally, model collision experiments by Beladjine et al. (2007) tend to show that the take-off velocity and angle are not correlated such that the bivariate distribution $P(\xi, \theta)$ can be expressed as the product of $P(\xi)$ and $P(\theta)$.

To conclude this section, we should emphasize that the picture of the splash process presented above is based on the description of a single collision event between an incident particle and a static bed. In contrast, when considering a equilibrium state of sand transport, several collision events may occur within a short time at a given surface area. It is generally assumed that collision events may be considered as a collection of single independent collision events. Most of the theoretical and numerical models make this assumption. The latter is probably justified for moderate wind speed but may break down for higher wind speed because the impact rate is an increasing function of the Shields parameter. A recent numerical work by Duran et al. (2014) 
mentions the importance of possible collective effects in the splash process. Besides, the hypothesis of a purely static bed may also be questioned: for high impact rates, we may expect that the bed surface is "fluidized".

\section{Numerical and Theoretical models}

In addition to wind-tunnel experiments, numerical and analytical studies have been carried out in attempts to understand and predict saltation. We first present approaches based on a Lagrangian description of the particle phase, where the particle trajectories are explicitely calculated and then briefly report on Eulerian approaches that have been developed.

\subsection{Lagrangian approaches}

\subsubsection{Model Equations}

Most approaches (Ungar and Haff, 1987; Anderson and Haff, 1988; Werner, 1990; Anderson and Haff, 1991; Kok and Renno, 2009; Creyssels et al., 2009) are based on the calculation of the trajectories of the grains which are determined by:

$$
\begin{aligned}
& \frac{d \boldsymbol{r}}{d t}=\boldsymbol{\xi}, \\
& \frac{d \boldsymbol{\xi}}{d t}=-\left(g+\frac{C \sqrt{g d}}{d \sigma} \xi_{z}\right) \hat{z}+\frac{C \sqrt{g d}}{d \sigma}\left(U-\xi_{x}\right) \hat{\boldsymbol{x}},
\end{aligned}
$$

where $\boldsymbol{r}=(x, z)$ and $\boldsymbol{\xi}=\left(\xi_{x}, \xi_{z}\right)$ are the particle position and velocity respectively and $C$ is the dimensionless nonlinear drag coefficient which includes both inertial and form drag (Creyssels et al., 2009). The air flow is usually described via a simplified hydrodynamic model based on the Prandtl mixing length theory,

$$
\frac{d U}{d z}=\frac{\sqrt{s_{a}(z) / \rho_{a}}}{\kappa l},
$$

where $s_{a}(z)$ is the local air borne shear stress at height $z, l=z$ is the mixing length and $\kappa=0.4$ is the Von Karman constant. The resolution of the problem thus consists in determining the vertical profile of the air borne shear stress, $s_{a}(z)$, during steady state saltation in order to be able to compute the equilibrium wind profile and the set of particle trajectories. In steady state transport, the total shear stress (i.e., the sum of the particle and air shear stress) is constant through the depth of the flow such that

$$
s_{a}(z)=\rho_{a}(\sigma-1) g d S^{*}-s_{p}(z),
$$

where $S^{*}$ is the dimensionless air shear stress in the particle-free gas (i.e., the Shields parameter) and $s_{p}$ is the particle shear stress. Neglecting collisions between particles within the flow, the latter is solely due to kinetic contributions:

$$
s_{p}(z)=-\rho_{p} \phi(z)<\xi_{x} \xi_{z}>,
$$

where $<.>$ denotes an average over all particles at a given height. The particle shear stress can be estimated from the determination of the particle trajectories which requires the knowledge of the equilibrium wind profile. However, as seen above, the determination of the equilibrium wind 
profile requires the knowledge of the particle shear stress so the features of the particle and fluid phase are coupled and the resolution of the problem is not straightforward.

Before going further, we would like to emphasize that the determination of the particle trajectories requires the knowledge of the wind profile as well as the particle velocity distribution at the bed. The latter depends strongly on the behavior of the particles near the bed which includes information on the splash. Theoretical and numerical models should therefore incorporate the description of this process to predict realistic features of the saltation transport.

\subsubsection{Standard Lagrangian approach}

In Lagrangian approaches based on the explicit calculation of the particle trajectories (Anderson and Haff, 1988; Werner, 1990; Anderson and Haff, 1991; Kok and Renno, 2009), the steady state of saltation is determined by an iterative procedure. Trajectories of particles with a range of initial velocities at the bed are followed until the particles return to the bed by integrating Eqs. 16 and 17 and assuming an unperturbed initial logarithmic wind profile. Simultaneously, the evolution of the distribution of particle velocities with height is calculated thus permitting the determination of the profiles of particle concentration and particle shear stress, calculated as averages (cf. Eq. 20), from it. The average local value of the particle shear stress is then used to update the wind profile using a mixing length assumption (cf. Eqs. 18 and 19). At the bed, the velocity distribution of the incoming particles is related to that of the outgoing particles through the splash function (cf previous section) so that a new set of initial velocities at the bed and a new set of trajectories can be calculated. The whole process is iterated until a steady state is reached.

Werner (1990) was one of the firsts to develop such a discrete model using a splash function derived from experiments on impacts of grains on a bed. Kok and Renno (2009) developed more recently a refined version of Werner's model that is able to account for transport of a mixture of grains of various sizes. To do this, they implement a splash function extended to polydisperse beds. These discrete simulations have the capacity to reproduce the profile average particle and air velocities and particle concentration measured in the laboratory and in the fields. In particular, in their simulations, as conjectured by Bagnold (1941), Kok and Renno (2009) found that the air profiles obtained over a range of wind speeds exhibited a focus point, above which their slope increased with increasing wind speed and, below which, their slope decreased with increasing wind speed. A consequence of this is that the residual air shear stress at the bed decreases with increasing wind speed. The latter is on the order of the dynamic threshold Shields parameter, $S_{d}^{*}$, for moderate Shields parameters (i.e., $S^{*}<5 S_{d}^{*}$ ), and vanishes at large Shields parameters. These discrete simulations (Kok et al., 2012) also predict that the grain speed at the bed surface is invariant with the wind strength in agreement with wind tunnel experiments (Creyssels et al., 2009; Ho et al., 2011).

\subsubsection{Other Lagrangian approaches}

Other Lagrangian approaches have been developed in the last decade. One can cite the numerical approach of Creyssels et al. (2009) which consists in solving the same equations as those of the Werner's model, but explicitely incorporates low-velocity moments of the splash function in a calculation of the boundary conditions that apply at the bed. More precisely, the average and the first velocity moment of the splash function are employed to calculate the average exchange of particles and particle momentum at the surface of the bed in steady conditions. For this, the velocity distribution of the ejected particles is assumed to be half-Gaussian. Two of the three parameters that characterize the velocity distribution function are determined by the balance of mass and vertical momentum between the flow and the bed. The remaining parameter is solved 
for in a simple and rapid numerical simulation of particles with a half-Gaussian distribution of initial vertical velocities interacting with and influencing the turbulent shearing flow before returning to the bed. This simple numerical simulation is found to reproduce most of the measured features of the flow of both the gas and the particles. These include the shape of the profiles of the average gas and grain velocities, the exponential decay of the concentration and the measured dependence of the total particle flux on the Shields parameter. The fact that a numerical simulation that employs a single free parameter reproduces the observed features of steady saltation opens the possibility of developing a better understanding of the process.

Among the recent Lagrangian approaches, one can also mention those based on discrete element methods (D.E.M.) (Duran et al., 2011; Carneiro et al., 2011, 2013). In contrast to the previous approaches, D.E.M. permit to resolve simultaneously the motion of the grains and their interactions including inter-particle collisions and fluid drag forces. No splash function is therefore required. DEM approaches are costly in terms of CPU times but present a powerful tool. As reported in Duran et al. (2011, 2012), DEM simulations have the capacity to predict the observed features of particle concentration of velocity profiles as well as the particle velocity distributions.

Finally, it is worthwhile to mention successful attempts to simplify the above Lagrangian approaches (Andreotti, 2004; Jenkins and Valance, 2014). In these simplified approaches, the distribution of the particle trajectories is replaced by either a single particle mode (Jenkins and Valance, 2014) ('one-species model') or two modes (Andreotti, 2004) including a weakly and highly energetic mode corresponding to the fast impacting particles and the slow splashed particles respectively ('two-species model').

\subsection{Eulerian (or continuum) approaches}

Eulerian models (Sauermann et al., 2001; Jenkins et al., 2010; Lämmel et al., 2012; Pähtz et al., 2012) are alternative and complementary approaches. In contrast to Lagrangian approaches, they do not require the explicit calculation of the particle trajectories but are based instead on the description of the particle phase as a continuum characterized by a local average particle velocity and concentration. These continuum approaches can also provide reasonable estimates of the salient features of the aeolian sand transport, including trajectory parameters. In addition, they provide an essential tool for modeling large scale phenomena such as ripple and dune formation.

In the present review there is no space for a detailed presentation of the different continuum models. We refer the reader to e.g. the recent continuum approaches developed by Jenkins et al. (2010), Lämmel et al. (2012) and Pähtz et al. (2012). However, we would like to address an important issue concerning these continuum approaches: what is the best strategy to parameterize the large distribution of particle trajectories and velocities with a reduced number of continuum variables? The simplest way (but not necessarily the most pertinent strategy) is to consider only the first moment of the particle velocity distribution as done in Sauermann et al. (2001). The further step is to include higher moments of the velocity distribution (e.g., the second moment known as the "granular" temperature) as developed in (Jenkins et al., 2010; Pähtz et al., 2012). An alternative approach for better parameterizing the particle velocity distribution is to consider two effective species of reptating and saltating grains (corresponding to weakly and highly energetic particles, respectively) as originally introduced by Bagnold (Bagnold, 1941). This approach has been recently developed by Lämmel et al. (2012) and may be a promising strategy to treat unsteady or inhomogeneous flow regimes of transport (see next section). The issue raised by this approach is to assign physical pertinent properties to each species. This is a matter of debate. 


\section{Unsteady and inhomogeneous transport}

Up to now, we have focused exclusively on the description of the equilibrium state of transport (i.e., steady and fully developed flow regime) where erosion and deposition processes balance exactly. A further interesting and important issue is the description of the out-of-equilibrium transport dynamics when the system is brought out of its equilibrium state. Let us imagine for example that the wind suddenly accelerates (see Fig. 11); the mass flow rate does not adapt instantaneously to its new equilibrium value. The system needs a certain time (or equivalently a certain distance) in order that the new grains extracted from the bed (initially at rest) equilibrate their velocity with that of the wind and that the particle concentration relaxes toward its new equilibrium value. During this transient process, the wind speed within the transport layer has to decrease to its equilibrium value because of the negative feedback of transport on the wind. The characteristic time and length scales of this relaxation process are usually referred to as the saturation time $\left(\tau_{\text {sat }}\right)$ and the saturation length $\left(l_{\text {sat }}\right)$, respectively (Sauermann et al., 2001; Andreotti et al., 2010; Pähtz et al., 2013).

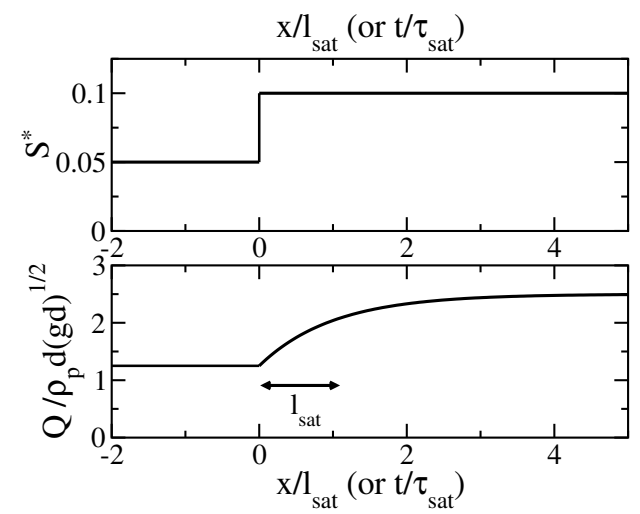

Figure 11: Spatial (or temporal) variation of the mass flow rate $Q$ over a sand bed following an increase of the wind speed: the mass flow rate relaxes to its new saturation value within a saturation length $l_{\text {sat }}$.

The saturation length is a priori driven by several intricate mechanisms for the wind speed, the particle velocity and the particle concentration to reach their equilibrium values. Several length scales may be a good candidate for the saturation length: the length needed for the negative feedback of transport on the wind to take place (Andreotti, 2004), the length needed to expel new grains from the sand bed (Sauermann et al., 2001), the saltation hop length $l_{s}$, and the length needed to accelerate new grains (called the drag length $l_{\text {drag }}$ (Hersen et al., 2002)) ${ }^{3}$. Importantly, the relaxation is limited by the slowest of these processes.

Experimental estimations of the saturation length by Andreotti et al. (2010) indicate that it is independent of the wind strength and is about twice the drag length: $l_{\text {sat }}=2 l_{\text {drag }}$ (for $d=200 \mu \mathrm{m}$, the saturation length is on the order of half a meter). These data therefore support the idea that grain inertia is the dominant dynamical mechanism limiting sediment transport saturation.

\footnotetext{
${ }^{3}$ The drag length is distance for a particle (initially at rest) to equilibrate its velocity with that of the fluid. It is controlled by the particle inertia and is given by $l_{\text {drag }}=\left(\rho_{p} / \rho_{a}\right) d$.
} 
It is important to stress that the saturation process is an essential feature for the formation of sand dunes (see the article review "Morphodynamics of dunes" by Sylvain Courech Du Pont). The saturation length actually controls the initial size of sand dunes emerging from a flat sand bed.

\section{Some open issues}

In this short review, we have depicted the present knowledge of the physical mechanisms controlling aeolian transport at the grain scale and at the scale of the transport layer. We have now a coherent picture of the equilibrium state of saltation transport on a flat and horizontal bed of monodisperse spherical particles. In this last section, we list several important issues that require further research.

An important issue is the dependence of the key features of the saltation transport (e.g., saltation layer height, particle velocity and concentration, saltation length, mass flux) upon the grain diameter. Theoretical and numerical approaches (Creyssels et al., 2009; Duran et al., 2011, 2012; Jenkins and Valance, 2014) predict in particular that the saltation layer height and the saltation length should scale linearly with the grain diameter. This linear scaling with the grain diameter is not confirmed by wind-tunnel experiments. Comparison between theoretical predictions and experimental data is however biased by the fact that theoretical approaches assume monodisperse spherical particles while experiments are usually carried out with natural sand samples whose grains are not perfectly spherical and exhibit a rather large size distribution (even for sieved samples). The additional experimental difficulty is to use different sand samples varying the median diameter while preserving the other features such as the shape, the size dispersion and the mechanical properties. There is therefore a need for additional wind-tunnel experiments where the heterogeneity of the sand particles in terms of size and sphericity is better controlled and characterized.

Another important issue is the role of the mid-air collisions. Most theoretical and numerical approaches neglect mid-air collisions. This assumption is probably pertinent for moderate Shields parameters but one expects mid-air collisions to play a prominent role at higher Shields parameters. The transition between pure saltation (without mid-air collisions) and sheet flow (predominance of mid-air collisions) is poorly documented. There exist only a few theoretical and numerical approaches (Pasini and Jenkins, 2005; Carneiro et al., 2013) but no experimental data.

Another issue concerns the influence of the presence of ripple patterns on the saltation transport. A flat and horizontal bed represents an idealized situation which rarely occurs in natural environment. The latter is indeed unstable with respect to the formation of sand ripples. Experimental and numerical results presented in this review concern exclusively transport over a flat and horizontal bed. There is a need for further numerical and experimental investigations to document the modification of the saltation transport over a rippled sand bed. A recent numerical work by Duran et al. (2014) reports a resonant mechanism between the saltating trajectories and a rippled bed.

A last important issue is the role of turbulent fluctuations. One specific problem in aeolian transport is the difficulty to compare controlled wind-tunnel experiments and field behavior. There is indeed a difference concerning the integral turbulent time-scale (Duran et al., 2011): in wind-tunnels it is of the order of a few seconds and corresponds to the transport time scale while in the field it is $10^{3}$ larger. If the nature of the turbulent fluctuations play a minor, as assumed in most the theoretical and numerical approaches, one can safely compare experimental 
and fields data when averaged over the correct time-scale. However, there are some situations where turbulent fluctuations are expected to play a major role, for example close to the static threshold or at high Shields parameter when saltation is replaced by intermittent saltation and turbulent suspension. Besides, specific issues concerning the structure of the turbulent flow (e.g. vortex dynamics) arise from field observations. Aeolian sand transport in natural environments exhibits complicated spatio-temporal transport patterns (not observed in wind-tunnels) called aeolian streamers which are ribbons of wind-blown sand in the stream-wise direction (Baas and Sherman, 2005). Further investigations are required to understand properly the role of the turbulent flow structure in the field.

\section{Acknowledgment}

We would like to thank K. Kroy and P. Clauding for their critical reading of the manuscript.

\section{References}

Anderson, R., Sørensen, M., Willetts, B., 1991. A review of recent progress in our understanding of aeolian sediment transport. Acta Mechanic Supply, 1, 1-19.

Anderson, R.S., Haff, P.K., 1988. Simulation of aeolian saltation. Science 241, 820-823.

Anderson, R.S., Haff, P.K., 1991. Wind modification and bed response during saltation of sand in air. Acta Mechanic Supply, 1, 21-51.

Andreotti, B., 2004. A two-species model of aeolian sand transport. Journal of Fluid Mechanics 510, 47-70.

Andreotti, B., Claudin, P., Pouliquen, O., 2010. Measurements of the aeolian sand transport saturation length. Geomorphology 123, 343-348.

Baas, A., Sherman, D., 2005. Formation and behavoir of aeolian streamers. Journal of Geophysical Research 110 , F03011.

Bagnold, R.A., 1941. The physics of blown sand and desert dunes. Methuen, New York

Beladjine, D., Ammi, M., Valance, A., Oger, L., 2007. Collision process between an incident bead and a threedimensional granular packing. Physical Review E. 75.

Carneiro, M.V., Araujo, N., Pähtz, T., Herrmann, H.J., 2013. Midair collisions enhance saltation. Phys. Rev. Lett. 111, 058001.

Carneiro, M.V., Pähtz, T., Herrmann, H.J., 2011. Jump at the onset of saltation. Phys. Rev. Lett. 107, 098001

Claudin, P., Andreotti, B., 2006. A scaling law for aeolian dunes on mars, venus, earth, and for subaqueous ripples. Earth and Planetary Science Letters 252, 30-44.

Crassous, J., Beladjine, D., Valance, A., 2007. Impact of a projectile on a granular medium described by a collision model. Physical Review Letters 99.

Creyssels, M., Dupont, P., Ould el Moctar, A., Valance, A., Cantat, I., Jenkins, J., Pasini, J., Rasmussen, K., 2009. Saltating particles in a turbulent boundary layer : experiment and theory. Journal of Fluid Mechanics 625, 47-74.

Dong, Z., Liu, X., Wang, X., Li, F., Zhao, A., 2004. Experimental investigation of the velocity of a sand cloud blowing over a sandy surface. Earth Surface Processes and Landforms 29, 343-458.

Dong, Z., Wang, H., 2004. Height profile of particle concentration in an aeolian saltating cloud: A wind tunnel investigation by piv msd. Geophysical Research Letter 30, 19, 10.1029/2003GL017915.

Duran, ., Claudin, P., Andreotti, A., 2011. On aeolian transport: Grain-scale interactions, dynamical mechanism and scaling laws. Aoelian Research 3, 243-270.

Duran, O., Andreotti, B., Claudin, P., 2012. Numerical simulation of turbulent sediment transport from bed load to saltation. Phys. Fluids 24, 103306.

Duran, O., Andreotti, B., Claudin, P., 2014. Direct numerical simulations of aeolian sand ripples. PNAS 111, 1566515668.

Greeley, R., Blumberg, D., S.H., W., 1996. Field measurement of the flux and speed of wind blown sand. Sedimentology 43, 41-52.

Hersen, P., Douady, S., Andreotti, B., 2002. Relevant length scale for barchan dunes. Physical Review Letters 89 264301.

Ho, T., 2012. Experimental study of saltating particles in a turbulent boundary layer. Ph.D. thesis. University of Rennes 1. 
Ho, T.D., Valance, A., Dupont, P., Ould El Moctar, A., 2011. Scaling laws in aeolian sand transport. Physical Review Letters 106, 094501.

Ho, T.D., Valance, A., Dupont, P., Ould El Moctar, A., 2012. Particle velocity distribution in saltation transport. Physical Review E

Ho, T.D., Valance, A., Dupont, P., Ould El Moctar, A., 2014. Aeolian sand transport: Height and length distribution of saltation trajectories. Aeolian Research , 65-74.

Iversen, J., Rasmussen, K., 1999. The effect of wind speed and bed slope on sand transport. Sedimentology 46, 723-731.

Jenkins, J., Cantat, I., Valance, A., 2010. Continuum model for steady, fully developed saltation above a horizontal particle bed. Physical Review E 82, 020301R.

Jenkins, J., Valance, A., 2014. Periodic trajectories in aeolian sand transport. Physics of Fluid 26, 073301.

Kok, J., Partel, E., Michael, T., Bou Karam, D., 2012. The physics of wind-blown sand and dust. Report on progress in Physics 75, 106901

Kok, J., Rennó, N., 2009. A comprehensive numerical model of steady state saltation. Journal of Geophysical Research. Atmospheres 114, D17204.

Lämmel, M., Rings, D., Kroy, K., 2012. A two-species continuum model for aeolian sand transport. New J. Phys. 14 093037.

Mitha, S., Tran, M.Q., Werner, B.T., Haff, P.K., 1986. The grain-bed impact process in aeolian saltation. Acta Mechanica 63, 267-278.

Nalpanis, P., Hunt, J., Barrett, C., 1993. Saltating particles over flat beds. Journal of Fluid Mechanics 251, 661-685.

Namikas, S.L., Bauer, B.O., Sherman, D.J., 2003. Influence of averaging interval on shear velocity estimates for aeolian transport modeling. Geomorphology 53, $235-246$.

Oger, L., Ammi, M., Valance, A., Beladjine, D., 2005. Discrete element method to study the collision of one rapid sphere on $2 d$ and 3d packings. The European Physical Journal E 17, 467-476.

Owen, P.R., 1964. Saltation of uniform grains in air. J. Fluid Mech. 20, $225-242$

Pähtz, T., Kok, J.F., Herrmann, H.J., 2012. The apparent roughness of a sand surface blown by wind from an analytica model of saltation. New Journal of Physics 14, 043035.

Pähtz, T., Kok, J.F., Herrmann, H.J., 2013. Flux saturation length of sediment transport. Physical Review Letters 111, 218002.

Pasini, J., Jenkins, J., 2005. Aeolian transport with collisional suspension. Phil.Trans.R. Soc. A 363, 1625-1646.

Rasmussen, K., Iversen, J., Rautahemio, P., 1996. Saltation and wind-flow interaction in a variable slope wind tunnel. Geomorphology 17(1-3), 19-28.

Rasmussen, K.R., Sørensen, M., 2008. The vertical variation of particle speed and flux density in aeolian saltation Measurement and modeling. Journal of Geophysical Research 113, F02S12.

Rice, M.A., Willetts, B.B., McEwan, I.K., 1995. An experimental study of multiple grain-size ejecta produced by collisions of saltating grains with a flat bed. Sedimentology 42, 4, 695-706.

Rice, M.A., Willetts, B.B., McEwan, I.K., 1996. Observations of collisions of saltating grains with a granular bed from high-speed cine-film. Sedimentology 43, 1, 21-31.

Rioual, F., Valance, A., Bideau, D., 2000. Experimental study of the collision process of a grain on a two-dimensional granular bed. Physical Review E. 62, 2.

Sauermann, G., Kroy, K., Herrmann, H.J., 2001. Continuum saltation model for sand dunes. Physical Review E. 64 031305.

Sherman, D., 1992. An equilibrium relationship for shear velocity and apparent roughness length in aeolian saltation. Geomorphology 5(3-5), 419-431.

Sørensen, M., 2004. On the rate of aeolian sand transport. Geomorphology 59, 53-62.

Ungar, J., Haff, P.K., 1987. Steady state saltation in air. Sedimentology 34, 289-299.

Werner, B.T., 1990. A steady-state model of wind-blown sand transport. Journal of Geology 98, 1-17.

Werner, B.T., Haff, P.K., 1988. The impact process in aeolian saltation: two-dimensional simulations. Sedimentology 35, 189-196.

White, B.R., Schulz, J.C., 1977. Magnus effect in saltation. Journal of Fluid Mechanics 81, 3, 497-512.

Willetts, B.B., Rice, M.A., 1986. Collisions in aeolian saltation. Acta Mechanica 63, 255-265.

Willetts, B.B., Rice, M.A., 1988. Particle dislodgment from a flat bed by wind. Earth Surface Processes and Landforms $13,717-728$

Willetts, B.B., Rice, M.A., 1989. Collision of quartz grains with a sand bed: the influence of incident angle. Earth Surface Processes and Landforms 14, 719-730.

Zhang, W., Kang, J., S., L., 2007. Tracking of saltating sand trajectories over a flat surface embedded in an atmospheric boundary layer. Geomorphology 86, 320-331. 